\title{
Metabolic approaches to antihypertensive treatment in diabetic patients
}

\author{
Arrigo F Cicero and Giuliano Tocci \\ Hypertension Research (2015) 38, 802-803; doi:10.1038/hr.2015.105; published online 1 October 2015
}

$\mathrm{H}_{\mathrm{i}}$ ypertension management and control is still a major clinical challenge. Despite the availability of modern diagnostic noninvasive options and effective, sustained and well-tolerated pharmacological strategies, observational studies and national registries have being continuously reported relatively poor rates of blood pressure (BP) control at both national and local levels. Several potential reasons can be proposed to try to explain this relatively failure of antihypertensive strategies, worldwide. ${ }^{1}$ Among these, one of the most appropriate seems to be the substantially high additional cardiovascular risk profile that very often characterizes patients with essential hypertension, being mostly due to the concomitant presence of metabolic abnormalities and markers of organ damage. These factors have proven to negatively affect physicians' abilities to achieve the recommended BP targets, particularly in a setting of daily clinical practice. ${ }^{2}$ Another valid reason may be related to potential risk of developing drug-related adverse events during the long course of antihypertensive therapy over the years, especially in patients already affected by diabetes, hypercholesterolemia, hypertriglyceridemia and other metabolic abnormalities. $^{3}$

To try to overcome these stockholders and to improve the overall rate of $\mathrm{BP}$ control in treated hypertensive patients, European guidelines have proposed a more systematic of effective, synergic and well-tolerated

AF Cicero is at Department of Medical and Surgical Sciences, Sant'Orsola Malpighi, University of Bologna, Bologna, Italy; G Tocci is at Division of Cardiology, Department of Clinical and Molecular Medicine, Faculty of Medicine and Psychology, University of Rome 'Sapienza', Sant'Andrea Hospital, Rome and IRCCS Neuromed, Pozzilli (IS), Italy

E-mail: arrigo.cicero@unibo.it combination therapies, based on the use of two or three pharmacological agents able to provide greater systolic/diastolic BP reductions than each component used in monotherapy and possibly additional, pleiotropic beneficial effects on metabolic parameters. In particular, current guidelines recommended the use of combination therapies based on renin-angiotensin system blocking agents, including either angiotensinconverting enzyme inhibitors or angiotensin receptor blockers (ARBs), plus dihydropyridinic calcium-channel blockers (CCBs), which have demonstrated to produce effective and sustained BP reductions, reduced incidence of major cardiovascular events, several metabolic advantages (for example, reduced incidence of new onset diabetes and improved lipid profile) with optimal tolerability profile, compared with other combination strategies. ${ }^{4}$

In the previous issue of the Hypertension Research, Derosa and co-workers analyzed the effects of two different and largely used combination therapies, one based on the ARB telmisartan plus hydrochlorothiazide and the other one based on the ARB losartan plus the CCB barnidipine, on some glucose and metabolic parameters, mostly including insulin sensitivity. ${ }^{5}$ The main findings of this study are of potential clinical relevance for several practical reasons. First of all, both telmisartan and losartan have proven to provide metabolic advantages in treated hypertensive patients at high or very high cardiovascular risk included in randomized clinical trials and both of them have demonstrated to reduce new incidence of diabetes mellitus, mostly throughout their selective peroxisome proliferator-activated receptor- $\gamma$ (PPAR- $\gamma$ ) stimulating properties. ${ }^{6}$ Secondly, both these strategies are currently recommended by guidelines to be used in hypertensive patients at metabolic risk or in those with metabolic abnormalities, to prevent development or reduce progression toward diabetes and its complications. ${ }^{7}$ Finally, the proposed strategies are also the one most used in clinical practice. ${ }^{8}$

At the end of the observation period, both treatments produced effective BP reductions, which are mandatory for any antihypertensive strategy, but systolic/diastolic BP levels were lower in losartan/barnidipine arm compared with those observed in telmisartan/ hydrochlorothiazide arm. Also, BP levels were in the normal range only in the former group compared with the latter group, which showed high-normal/above normal BP levels. These results were associated with significant and beneficial metabolic effects, which were not related to BP reductions. From one side, contrarily to what it could have been expected no improvement in insulin-resistance has been observed, however this could be related to the fact that the enrolled patients had a perfect control of their diabetes at the baseline. On the other side, it is of relevant interest the plausible effect of barnidipine on adipokines level, that could have relevant effect on endothelial function and, consequently, on vascular health of diabetic patients. In fact, it is well-known that, with respect to cardiovascular disease risk, adiponectin is a 'good' adipokine due to its antiinflammatory, antiatherogenic, antidiabetic, cardioprotective effects and promotion of good endothelial function. Leptin, on the contrary, it could be considered a 'bad' adipokine given that it promotes insulin resistance, high $\mathrm{BP}$, atherosclerosis, myocardial infarction risk, vascular inflammation, vascular smooth muscle cell hypertrophy and proliferation, oxidative stress and endothelial 
dysfunction. $^{9}$ The cardiovascular effects of other adipokines in humans are yet to be fully clarified.

It could be also argued that these results are at least partly magnified by the comparison of a thiazide that could have some negative metabolic effects. ${ }^{10}$ However, in this trial hydrocholorthiazide was used at low dosage, usually not associated to a significant impairment in metabolic parameters, while it was associated to a more efficacious activator of the PPAR- $\gamma$ (telmisartan), ${ }^{6}$ that theoretically should have compensated the eventual mild negative effects of the thiazide. Finally, it has to be remembered that telmisartan could also exert specific anti-inflammatory effects, ${ }^{11,12}$ as demonstrated in a number of clinical trials, which could be relevant in the long-term treatment of hypertension in high-risk subjects. In any case, the lack of negative effects on lipid pattern and glucose metabolism confirms that the used doses of hydrochlorothiazide were unable to exert any side effect in the studied patients.

Anyway, adequately powered long-term trials have to be planned and carried out to verify if the observed hemodynamic and metabolic effects of the association losartan/ barnidipine could be associated to relevant clinical outcome such as target organ damage or cardiovascular event prevention.

\section{CONFLICT OF INTEREST}

The authors declare no conflict of interest.

1 Lemogoum D. Challenge for hypertension prevention and control worldwide: the time for action. J Clin Hypertens 2014; 16: 554-556.

2 Borghi C, Cicero AF. How to avoid the depression of the adherence to antihypertensive treatment? J Hypertens 2013; 31: 1362-1363.

3 Cicero AF, Ertek S. Hypertension and diabetes incidence: confounding factors. Hypertens Res 2011; 34: 1069-1070.

4 ESH/ESC Task Force for the Management of Arterial Hypertension. Practice guidelines for the management of arterial hypertension of the European Society of Hypertension (ESH) and the European Society of Cardiology (ESC): ESH/ESC Task Force for the Management of Arterial Hypertension. J Hypertens 2013; 31: 1925-1938.

5 Derosa G, Querci F, Franzetti I, Ragonesi PD, D'Angelo A, Maffioli P. Comparison of the effects of barnidipine+losartan compared to telmisartan+hydrochlorothiazide on several parameters of insulin sensitivity in patients with hypertension and type 2 diabetes mellitus. Hypertens Res 2015; 38: 690-694.

6 Michel MC, Foster C, Brunner HR, Liu L. A systematic comparison of the properties of clinically used angiotensin II type 1 receptor antagonists. Pharmacol Rev 2013; 65: 809-848.

7 Borghi C, Cicero AF. Hypertension: management perspectives. Expert Opin Pharmacother 2012; 13: 1999-2003.

8 Tocci G, Cicero AF, Salvetti M, Francia P, Ferrucci A, Borghi C, Volpe M. Attitudes and preferences for the clinical management of hypertension and hypertensionrelated cardiac disease in general practice: results of the Italian Hypertension and Heart Survey. J Hum Hypertens 2015; 29: 409-416.

9 Blüher M, Mantzoros CS. From leptin to other adipokines in health and disease: facts and expectations at the beginning of the 21st century. Metabolism 2015; 64: 131-145.

10 Price AL, Lingvay I, Szczepaniak EW, Wiebel J, Victor RG, Szczepaniak LS. The metabolic cost of lowering blood pressure with hydrochlorothiazide. Diabetol Metab Syndr 2013; 5: 35.

11 Takagi H, Mizuno Y, Yamamoto H, Goto SN, Umemoto T. All-Literature Investigation of Cardiovascular Evidence Group. Effects of telmisartan therapy on interleukin-6 and tumor necrosis factor-alpha levels: a meta-analysis of randomized controlled trials. Hypertens Res 2013; 36: 368-373.

12 Mikami D, Kimura H, Kamiyama K, Torii K, Kasuno K, Takahashi N, Yoshida H, Iwano M. Telmisartan activates endogenous peroxisome proliferator-activated receptor- $\delta$ and may have anti-fibrotic effects in human mesangial cells. Hypertens Res 2014; 37: 422-431. 